\title{
Frequency and Time-Domain Comparison between LCL and LLCL Filters for a Grid-Connected Inverter Using Selective Harmonic Modulation
}

\author{
Rawia Chakroun $(\mathbb{D}$, Ramzi Ben Ayed $(\mathbb{D}$, and Nabil Derbel $\mathbb{D}$ \\ Control \& Energy Management Laboratory (CEMLab), Digital Research Center of Sfax (CRNS), University of Sfax, \\ Sfax Engineering School, BP 1173, Sfax 3038, Tunisia \\ Correspondence should be addressed to Rawia Chakroun; rawiachakroun7@gmail.com
}

Received 26 January 2021; Accepted 14 June 2021; Published 23 June 2021

Academic Editor: Hao Shen

Copyright (C) 2021 Rawia Chakroun et al. This is an open access article distributed under the Creative Commons Attribution License, which permits unrestricted use, distribution, and reproduction in any medium, provided the original work is properly cited.

\begin{abstract}
This paper shows a comparison between the analytical design of a photovoltaic power station filter and a real-case filter. Indeed, the analytical filter considered in the design phase is an LCL filter, while the real case is an LLCL filter. This difference could impact the current harmonics rejected on the grid and breaks grid codes. The main objective of this study is to maximize the power injected into the network while respecting the harmonic rejection standards in force, namely, G5/4, French decree of 2008, and IEEE 519 standards, by adopting a current control design to improve the performance of a grid-connected three-phase inverter, which is regarded as the central component in a photovoltaic production system. The selective harmonic modulation strategy (SHM) is a common technique to achieve this goal. For that, a frequency and a time-domain comparison for a grid-connected inverter using both filters have been highlighted. Simulation results confirm the excellent transient behavior of both filter topologies and the advantage to consider the flexibility of LLCL filter when combined with SHM strategy. This manuscript is an extension of an earlier version of "Comparison between LCL and LLCL Filters for a Grid Connected Inverter Using Selective Harmonic Modulation.”
\end{abstract}

\section{Introduction}

The technological progress could make today renewable energy sources as a fascinating area filled with innovations. Specific attention has to be paid in photovoltaic energy sources which could be considered as an important research field due to their large invasion in recent years, especially for a photovoltaic grid-connected inverter. No one can deny the crucial role of an inverter in any solar energy device, that is why, it is often considered as the central component of a photovoltaic substation. Furthermore, its basic function is to convert the DC current output to AC current and then connect to a large grid via the filter and insulation transformer. Modulation strategy is at the heart of the inverter. Based on this background, a multitude of modulation techniques have been invented. Due to its versatility and ease of use with different objectives, the selective harmonic modulation (SHM) is a very attractive key for high-power applications. An LCL filter is often used to interconnect the inverter to the grid to filter the harmonics generated by the inverter, which must be minimized to the maximum extent possible in order to prevent interruption of the utility and to comply with the grid code standards G5/4, French Decree 2008, and IEEE 519 [1-3], at the common coupling point. The analysis of the LCL filter design technique has been strongly used in theory but not in practical fields. For that, it seems crucial to design a real-case LLCL filter. These two topologies are differentiated from each other by their diagram modeling, their frequency responses, and their time responses, which can impact the current harmonics rejected on the grid and break grid code requirements. Several strategies have been presented and discussed in the literature 
for the design of LCL and LLCL filters [4-6]. All of them have attempted to efficiently model LCL and LLCL filter parameters according to various constraints based on parameter specifications, whereas filter parameters of both filter topologies are imposed. Until now and after several years of research in this field, there has been no definitive model that accurately and precisely explains the effect that may exist within both filters. Furthermore, the resonant behavior of LCL and LLCL filters could significantly impact the current harmonics rejected on the grid and break grid codes. The considered design methodology incorporates the benefits of the existing design methodologies and seeks to avoid their drawbacks. The aim of this paper is to contribute as an advance to previous works, to avoid this negative impact. Moreover, it presents a better alternative to avoid several problems that may occur. Indeed, it highlights with a detailed comparative study, a current control model strategy for LCL and LLCL filters for grid-connected three-phase inverters to apply with grid code requirements. Through the simulation tests, the accuracy and viability of adopted strategies is well checked. This paper is organized as follows. In Section 2, the selective harmonic modulation principle is briefly summarized. In section 3 , the adopted selective harmonic modulation strategy is depicted. Section 4 describes the LCL filter design process as a theoretical study. The LLCL filter design process is well developed in Section 5. Simulation results and discussion are carried out in Section 6 . Finally, conclusions of this paper are presented in Section 7.

\section{Basic Principle of the SHM Strategy}

Based on the Fourier analysis of the conventional preprogrammed pulse width modulation switching pattern, we have the possibility to model the harmonic spectrum. This is possible thanks to the correlation established by Patel and Hoft [7], which links the position of the angle to the generated harmonic amplitude. Considering $k$, the number of switching angles, $n$ is the harmonic order, and the switching angle, $\alpha_{i}(i=1, \ldots, k)$, generates the following equations, where $T \bmod _{n}$, is the harmonic amplitude of the $n^{\text {th }}$ order:

$$
\operatorname{Tmod}_{n}=\frac{4}{n \pi}\left|1+2 \sum_{i=1}^{k}(-1)^{i} \cos \left(n \alpha_{i}\right)\right|
$$

where $n=5,7,11, \ldots, q$.

The core idea of the SHM strategy [8] is based on the fact that it is not important to reduce harmonics to zero while they are maintained below acceptable limits. These rates are specified by grid codes [1-3], that set the maximum allowed limits for each harmonic in order to maintain the performance of the grid. The SHM technique is focused on solving a series of inequalities where $\varepsilon_{q}$ is the maximum value specified by the grid code standards. The inequality framework can be synthesized in an objective function (OF) that must be minimized:

$$
\mathrm{OF}=\left(\operatorname{Tmod}_{1}-\mathrm{Md}\right)^{2},
$$

subject to

$$
\begin{aligned}
\operatorname{Tmod}_{5} & \leq \varepsilon_{5} \\
\operatorname{Tmod}_{7} & \leq \varepsilon_{7} \\
& \vdots \\
\operatorname{Tmod}_{q} & \leq \varepsilon_{q}, \\
0 & <\alpha_{1}<\cdots<\alpha_{i}<\alpha_{i+1}<\cdots<\alpha_{n}<\frac{\pi}{2},
\end{aligned}
$$

where $\mathrm{Md}$ is the desired modulation index.

This technique has the advantage of being able to deal with strongly nonlinear optimization problems.

\section{Adopted SHM Strategy}

In this paper, seven switching angles have been applied. Thus, only the six nontriple lower order harmonics, 5th, 7th, 11 th, 13th, 17th, and $19^{\text {th }}$, are controlled. To optimize the adopted SHM strategy and to present really suitable results, practical implementation constraints have been taken into account in the analysis. For instance, grid code standards authorize a maximum allowable limit for each harmonic current content. To apply these requirements, a deterministic optimization method is carried out, replacing each $\varepsilon_{q}$ factor with its respective harmonic current grid code value limit. Previous works only aim to comply with one or two grid standards, but in this case, we are attempting to meet the G5/4, French Decree 2008, and IEEE 519 specifications, in order to ensure that the photovoltaic substation performs well throughout the world. $\varepsilon_{q}$ will be set as the minimum level of G5/4, French Decree 2008, and IEEE 519. The SHM technique is based on solving a set of inequality system, where $\varepsilon_{q}$ are limit values defined by the applied grid code. The inequality system can be synthesized in an OF which has to be minimized, subject to

$$
\begin{gathered}
\operatorname{Tmod}_{5} \leq \varepsilon_{5}, \\
\operatorname{Tmod}_{7} \leq \varepsilon_{7}, \\
\operatorname{Tmod}_{11} \leq \varepsilon_{11}, \\
\operatorname{Tmod}_{13} \leq \varepsilon_{13}, \\
\operatorname{Tmod}_{17} \leq \varepsilon_{17}, \\
\operatorname{Tmod}_{19} \leq \varepsilon_{19}, \\
0<\alpha_{1}<\alpha_{2}<\alpha_{3}<\alpha_{4}<\alpha_{5}<\alpha_{6}<\alpha_{7}<\frac{\pi}{2}, \\
\alpha_{i+1}-\alpha_{i} \geq \theta_{i},
\end{gathered}
$$

where $\varepsilon_{5}, \varepsilon_{7}, \varepsilon_{11}, \varepsilon_{13}, \varepsilon_{17}$, and $\varepsilon_{19}$ are imposed by the grid code requirements. 


$$
\theta_{i}=\omega_{s} t_{d}=2 \pi f_{s} t_{d},
$$

where $\theta_{i}$ is the angle which corresponds to the dead-time constraint, $f_{s}$ is the switching frequency, and $t_{d}$ is the deadtime of the silicon IGBT. The dead-time $\left(t_{d}\right)$ is expressed as follows:

$$
t_{d}=t_{d_{\mathrm{on}}}+t_{r}+t_{d_{\mathrm{off}}}+t_{f}+t_{s}
$$

where

(i) $t_{d_{\mathrm{on}}}:$ turn-on delay time $(\mu \mathrm{s})$

(ii) $t_{r}$ : current rise time $(\mu \mathrm{s})$

(iii) $t_{d_{\text {off }}}:$ turn-off delay time $(\mu \mathrm{s})$

(iv) $t_{f}$ : current fall time $(\mu \mathrm{s})$

(v) $t_{s}$ : safety margin $(\mu \mathrm{s})$

On conventional silicon IGBT, the response time is around $5 \mu \mathrm{s}$ for switching on and $5 \mu \mathrm{s}$ for switching off.

$t_{s}$ corresponds to the safety margin which is equal to $5 \mu \mathrm{s}$.

Seven switching angles have been used in this paper, and this does not allow to comply with grid codes without any additional filtering system [9]. As the remaining harmonics are uncontrolled, that is why a passive filter is required.

\section{Electrical Scheme with a Theoretical LCL Filter}

Due to their fascinating contribution to grid-connected systems, LCL filters are increasingly being used in highpower applications today.

4.1. General Schematic Modeling of a Photovoltaic Substation with a Theorotical LCL Filter. Figure 1 shows the proposed photovoltaic system which contains the following:

(i) A photovoltaic array

(ii) A chopper to track MPPT and regulate the DC bus

(iii) A three-phase inverter that converts the DC voltage to $\mathrm{AC}$ voltage

(iv) A transformer to guarantee the galvanic isolation as the grid has a voltage of $25 \mathrm{kV}$ to amplify the voltage supplied

4.2. Equivalent Electrical Diagram Modeling. In our study case, the smoothing inductor of the filter is combined with the transformer. The combination of filter inductor and transformer allows to optimize the volume, the mass, and the cost. The electrical configuration of this model is shown in Figure 2. As depicted in this figure, the LCL filter is connected between the inverter and the grid through the transformer.

4.3. Equivalent Frequency Diagram Modeling. The singlephase equivalent circuit with a connected LCL filter is shown in Figure 3.

The equivalent circuit of a grid-connected three-phase inverter is an alternative voltage source which generates the

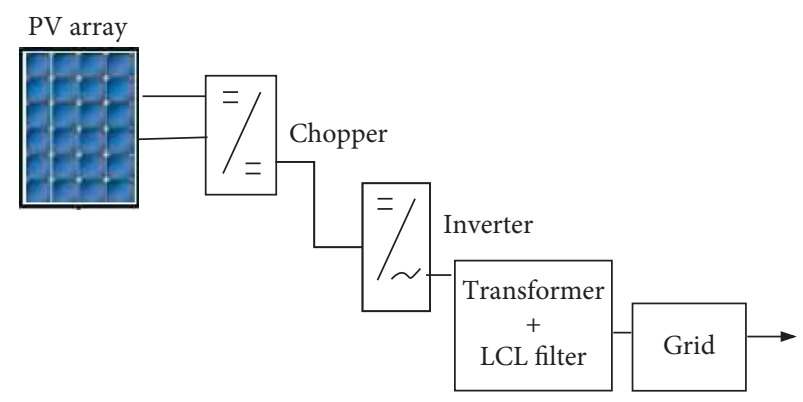

FIGURE 1: General schematic modeling of a photovoltaic substation with a theoretical LCL filter.

harmonics caused by the power inverter. The transformer, the filter, and grid parameters of the equivalent single-phase frequency diagram are well described as follows:
(i) $R_{2 Y 1}$ : secondary winding resistance $(\Omega)$
(ii) $L_{2 Y 1}$ : secondary inductance $(\mathrm{H})$
(iii) $R_{3 Y 1}$ : tertiary resistance $(\Omega)$
(iv) $L_{3 Y 1}$ : tertiary inductance $(\mathrm{H})$
(v) $C_{3 Y 1}$ : tertiary capacitance $(\mathrm{H})$
(vi) $R_{1 Y 1}$ : primary winding resistance $(\Omega)$
(vii) $L_{1 Y 1}$ : primary inductance $(\mathrm{H})$
(viii) $R_{\mu 1}$ : magnetic resistance $(\Omega)$
(ix) $L_{\mu 1}$ : magnetic inductance $(\mathrm{H})$
(x) $R_{\text {grid } 1}$ : equivalent grid resistance $(\Omega)$
(xi) $L_{\text {grid1 } 1}$ : equivalent grid inductance $(\mathrm{H})$

4.4. Mathematical Analysis of a Theoretical LCL Filter. For harmonic at high frequencies, the impedances of $L_{\mu 1}$ and $R_{\mu 1}$ are much greater than those of $L_{1 Y 1}, R_{1 Y 1}, R_{\text {grid1 }}$, and $L_{\text {grid1 } 1}$. Therefore, we remove $R_{\mu 1}$ and $L_{\mu 1}$ from the corresponding scheme. Likewise, the resistances, $R_{1 Y 1}, R_{2 Y 1}$, and $R_{\text {grid } 1}$ are negligible in front of the respective inductances. Under the above assumptions, the equivalent single-phase scheme is given in Figure 4.

As shown in Figure 4, the equivalent model is a T-type LCL filter which is composed from the secondary inductance $L_{2 Y 1}$, the equivalent grid inductance $L_{\text {grid1 }}$, and the equivalent tertiary $C_{3 Y 1}$ capacitor.

For the $n$-harmonic and neglecting the resistor effects, we deduce the amplitude of each harmonic of the current $i_{h}$ rejected as a function of the inverter voltage harmonic $V_{h}$ :

$$
i_{h}=\frac{V_{h}}{\left|\omega\left(L^{\prime}-\omega^{2} F\right)\right|},
$$

where

$$
\begin{aligned}
& L^{\prime}=L_{1 Y 1}+L_{2 Y 1}+L_{\text {grid1 }}, \\
& F=C_{3 Y 1} L_{2 Y 1}\left(L_{1 Y 1}+L_{\text {grid } 1}\right) .
\end{aligned}
$$

During the development phase, we realize that the cables that will be used to connect the transformer tertiary to the capacitors' bank introduce a leakage inductance which must 


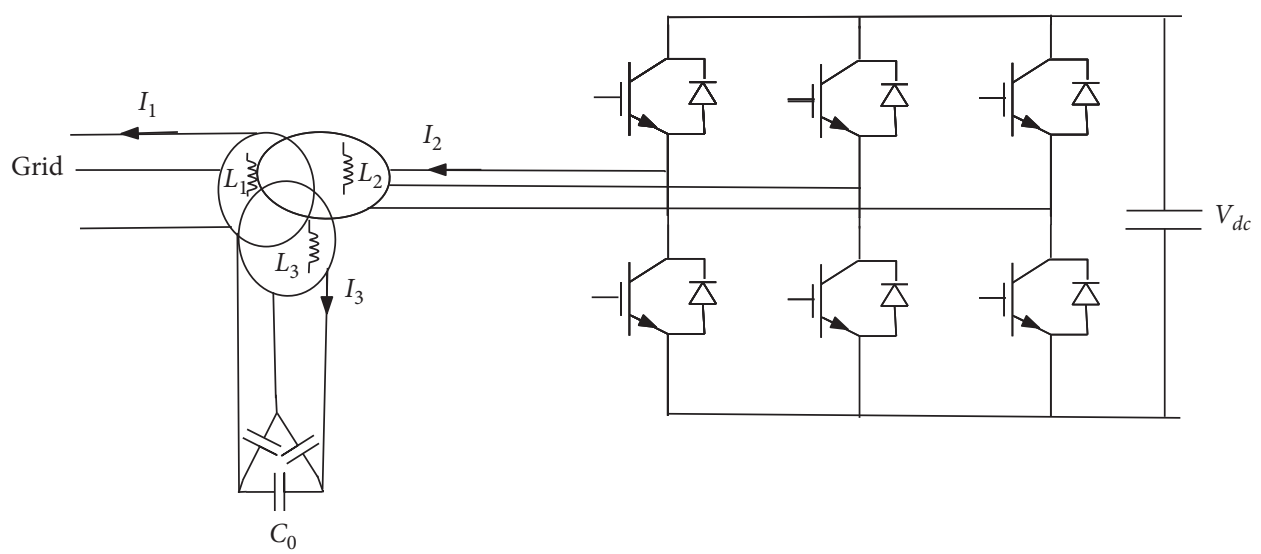

FIGURE 2: General power circuit of a three-phase grid-connected power converter with a theoretical LCL filter.

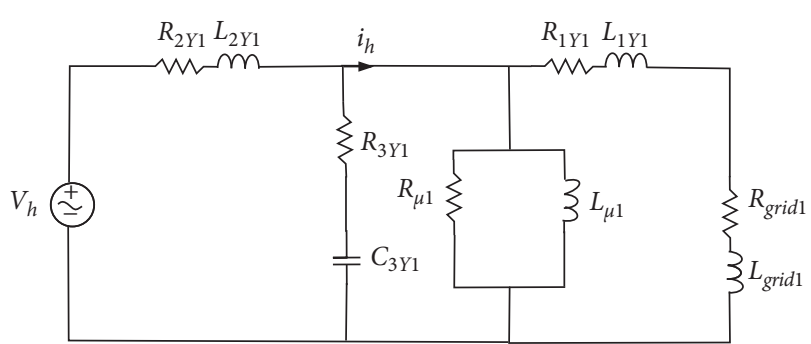

FIGURE 3: Per-phase equivalent circuit with a connected LCL filter.

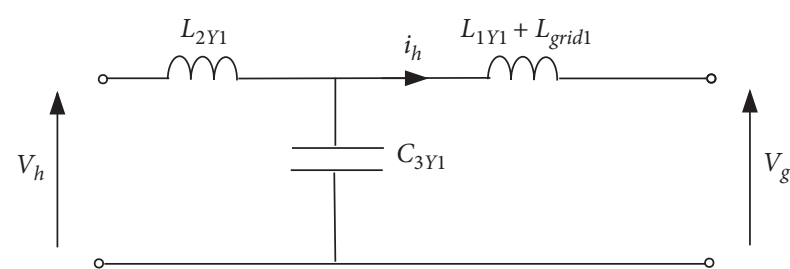

FIGURE 4: Equivalent single-phase representation of the threephase grid-connected power converter with LCL filter.

be taken into account. That leads to LLCL topology instead of LCL topology.

\section{Electrical Scheme with a Real-Case LLCL Filter}

5.1. General Schematic Modeling of a Photovoltaic Substation with a Real-Case LLCL Filter. The synoptis of the proposed $\mathrm{PV}$ system with an LLCL filter is given in Figure 5.

5.2. Equivalent Electrical Diagram Modeling. The same electrical scheme using the LCL filter is considered, but the leakage inductor related to the cable length has been added as shown in Figure 6. That makes the LLCL filter flexible as its behavior depends on the cable length which is optimized in [10]. The leakage inductor of a tertiary cabling is named $L_{3 Y 1}$.

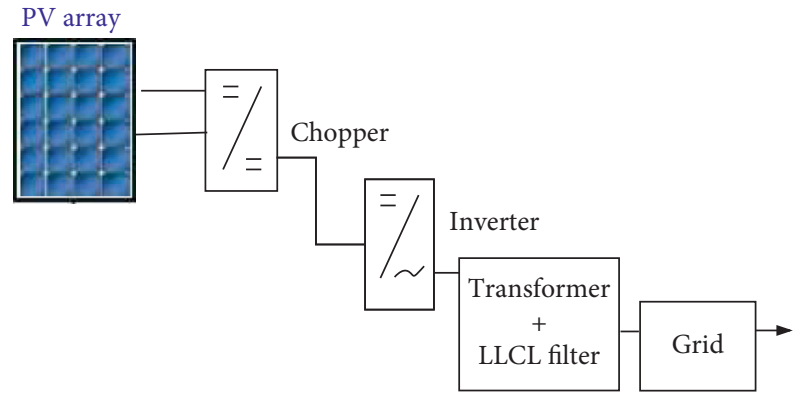

Figure 5: General schematic modeling of a photovoltaic substation with a real-case LLCL filter.

5.3. Equivalent Frequency Diagram Modeling of a Real-Case LLCL Filter. The single-phase equivalent circuit with a connected LLCL filter is shown in Figure 7 , where $L_{3 Y 1}$ is a tertiary inductance $(\mathrm{H})$.

Based on the same assumptions as the LCL filter case related to tertiary cabling, the single-phase equivalent diagram with a connected LLCL filter is presented in Figure 8.

5.4. Mathematical Analysis of a Real-Case LLCL Filter Design. Under the per-phase equivalent frequency diagram, we deduce the amplitude of each harmonic of the rejected current as a function of the inverter voltage harmonic:

$$
i_{h}=\frac{\left|1-\omega^{2} N\right| V_{h}}{\left|\omega\left(L^{\prime}-\omega^{2} D\right)\right|},
$$

where

$$
\begin{aligned}
& N=C_{3 Y 1} L_{3 Y 1}, \\
& L^{\prime}=L_{1 Y 1}+L_{2 Y 1}+L_{\text {grid1 }}, \\
& D=C_{3 Y 1}\left(L_{2 Y 1}+L_{3 Y 1}\right)\left(L_{1 Y 1}+L \text { grid } 1\right)+L_{2 Y 1} L_{3 Y 1} C_{3 Y 1} .
\end{aligned}
$$




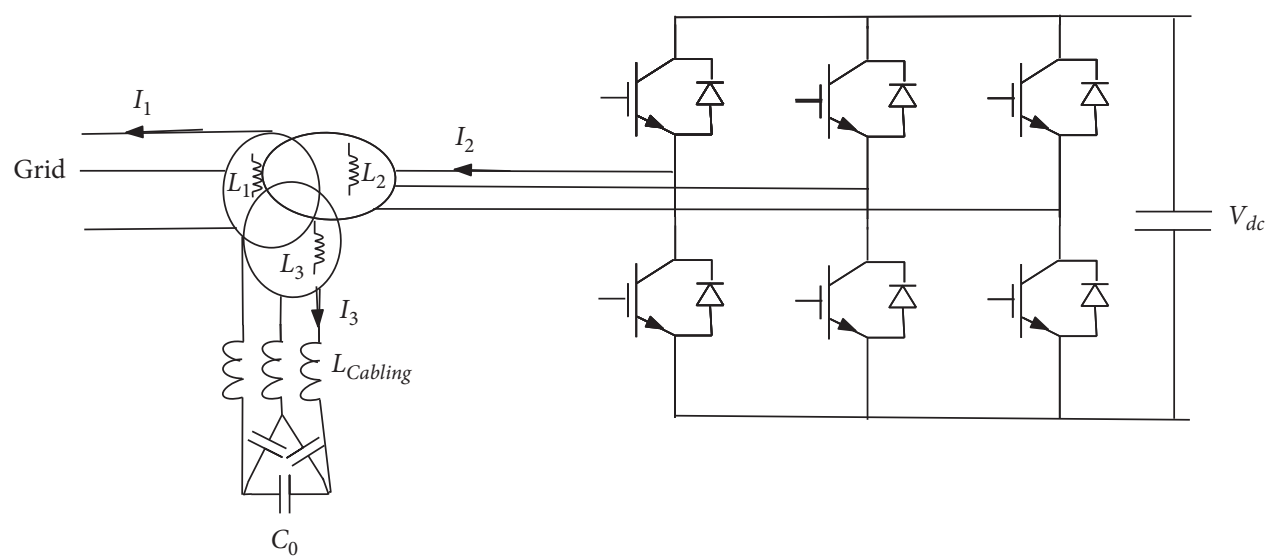

FIGURE 6: General power circuit of a three-phase grid-connected power converter with LLCL filter.

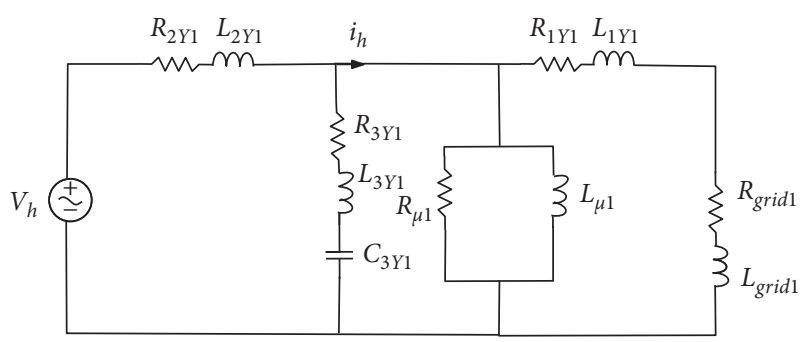

Figure 7: Per-phase equivalent circuit with a connected LLCL filter.

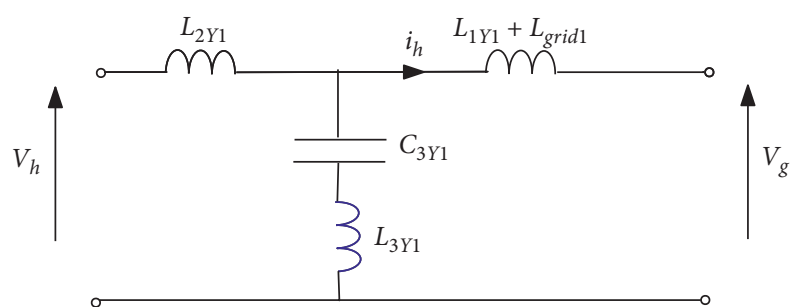

FIGURE 8: Equivalent per-phase frequency diagram modeling of a photovoltaic grid-connected inverter.

\section{Simulation Results and Discussion}

To verify the efficiency of the adopted SHM strategy, a detailed model of the grid-connected inverter as well as the investigated topologies is implemented in the simulation tool. The simulation work is meant to prove the robustness and to identify differences of both filters. Comparison between performances of LCL and LLCL filters under the same assumptions is carried out. In fact, factors taken into consideration while comparing their performances are based on their impact on the current harmonics rejected on the grid.

6.1. Implementation and Algorithm Validation. The use of an electrical system design optimization has become a common practical approach [11-14]. Several optimization strategies have been developed in the literature $[15,16]$. To validate the stability condition and to guarantee the convergence properties, a deterministic optimization based on the famous sequential quadratic programming (SQP) has been implemented. For each Md value from 0 to $(4 / \pi)$ by step of 0.01 , SQP is used to solve the optimization problem expressed in equations from (1) to (11). Then, for each Md value, we got the vector of the seven angles $\alpha_{i}$.

6.2. Extraction of the Desired Modulation Index, $M d$. In order to extract the desired modulation index value, a system of equations should be established. For that, an algorithm based on the fixed point iteration is built to obtain the desired modulation index. The obtained modulation index under the nominal working conditions is $\mathrm{Md}=0.86$.

6.3. Frequency Response for the LCL and the LLCL Filter Design. The transfer functions of LCL and LLCL filters are defined as the ratio between the output current $i_{h}$ and the input voltage $V_{h}$.

$$
\begin{aligned}
H_{\mathrm{LCL}} & =\frac{1}{p L^{\prime}+p^{3} F}, \\
H_{\mathrm{LLCL}} & =\frac{1+p^{2} N}{p L^{\prime}+p^{3} D},
\end{aligned}
$$

where $p=j \omega$.

In Figure 9, the characteristics of LCL and LLCL filters show a resonant behavior. Furthermore, in the low frequency range, the behavior of both filters is identical till the resonance. Besides, the LCL filter offers high attenuation at high frequencies, while this rate is limited for the LLCL real case. Thus, the theoretical LCL filter has a good harmonic attenuation and a sufficient stability margin. However, the LCL filter characteristic has one peak which corresponds to the resonance frequency, but the LLCL filter has two peaks in its transfer function. The second peak corresponds to the socalled antiresonance phenomenon. Between resonance and antiresonance, the LLCL filter shows better performances than the LCL filter. 


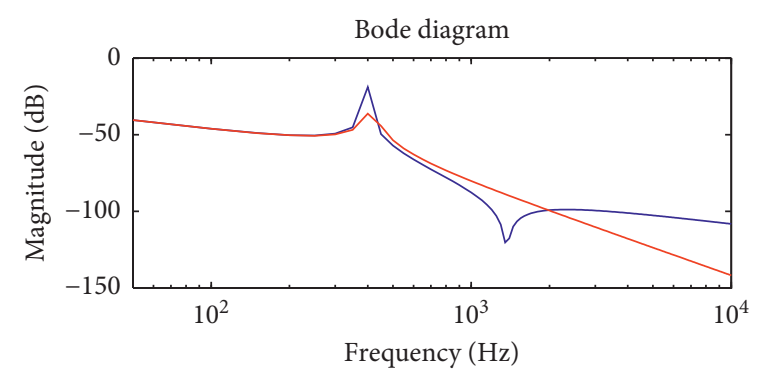

(a)

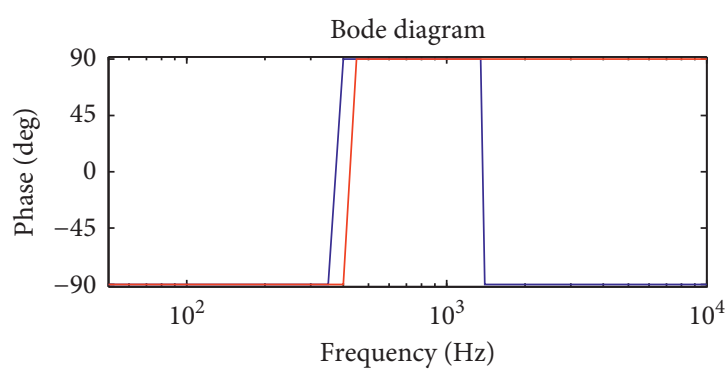

(b)

FIgUre 9: Bode plots of the transfer function for the LCL (in red) and LLCL filters (in blue) till $10 \mathrm{kHz}$.

6.4. Harmonic Spectrum with a Theoretical LCL Filter. In the computing process, the SHM technique has been used to determine switching angles that generate the most appropriate harmonic spectrum for a theoretical LCL filter.

Figure 10 shows the harmonic current amplitude for a theoretical LCL filter, as a function of the harmonic order. These results show that the six first harmonics are not eliminated but controlled by the SHM algorithm [9], while the remaining harmonics are attenuated by the LCL filter which shows very good attenuation at high frequencies. A real-case LLCL filter is illustrated in Section 6.5.

6.5. Harmonic Spectrum with a Real-Case LLCL Filter. Like for LCL filter case, SHM technique has been used to determine the switching angles.

Figure 11 shows the harmonic current amplitude for a real-case LLCL filter, as a function of the harmonic order. This figure for the LLCL filter shows that the first harmonics are not eliminated and remain controlled by the SHM algorithm like in the LCL filter case. However, the LCL filter shows better attenuation capability near the antiresonance peak but less capability of harmonics attenuation than LCL filter at high frequencies.

The compliancy of both spectra shown in Figures 10 and 11 with grid codes is shown and analyzed in the following sections.

6.6. LCL versus Grid Codes. In this section, we check the compliancy of SHM-LCL results with grid codes.

From the simulation results shown in Figure 12, it is obvious that the theoretical LCL filter successfully meet grid codes standard limits. This appreciable result confirms the efficiency of the LCL filter.

6.7. LLCL versus Grid Codes. Figure 13 shows the harmonic current amplitude with a real-case LLCL filter as a function of the harmonic order versus grid codes.

From the simulation results, it is clear that the real-case LLCL filter successfully meets grid codes limits. This appreciable result confirms the efficiency of the real-case LLCL filter.

Both figures show that SHM with LCL or LLCL filter leads to good results and comply with grid codes. This confirms what it has been found in the previous studies. In

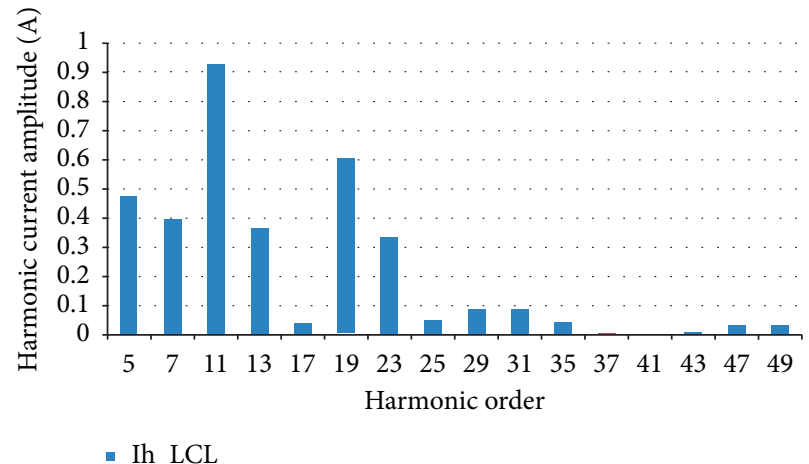

FIgURE 10: Harmonic current spectrum with a theoretical LCL filter.

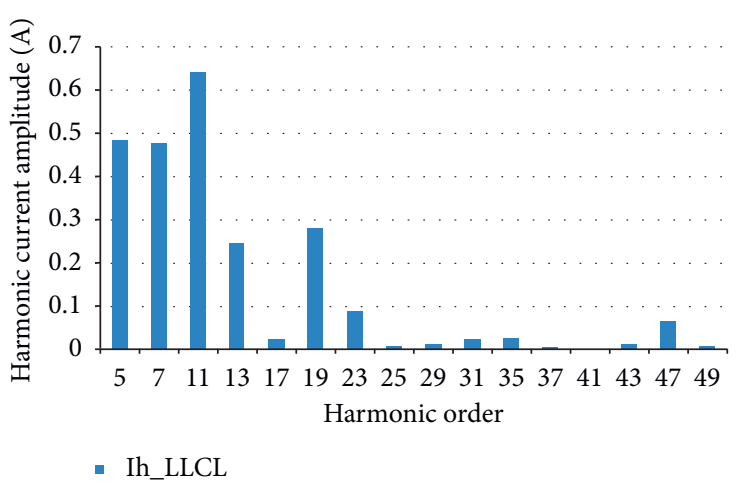

FIgURE 11: Harmonic current spectrum with a real-case LLCL filter.

order to understand the difference between both filters, both spectra are compared.

6.8. Time-Domain Comparison of SHM-LCL versus SHMLLCL. Figure 14 shows the output current curve obtained with the SHM-LCL strategy. The THD obtained with this strategy is around $6 \%$.

The current injected on the grid by the SHM-LLCL strategy is shown in Figure 15. The THD obtained with this strategy is around $4.5 \%$.

Both curves show satisfactory results as the THD is below 8\% (the most common THD tolerated on the grids worldwide). The SHM-LLCL strategy shows a much better THD than the SHM-LCL strategy that confirms that 


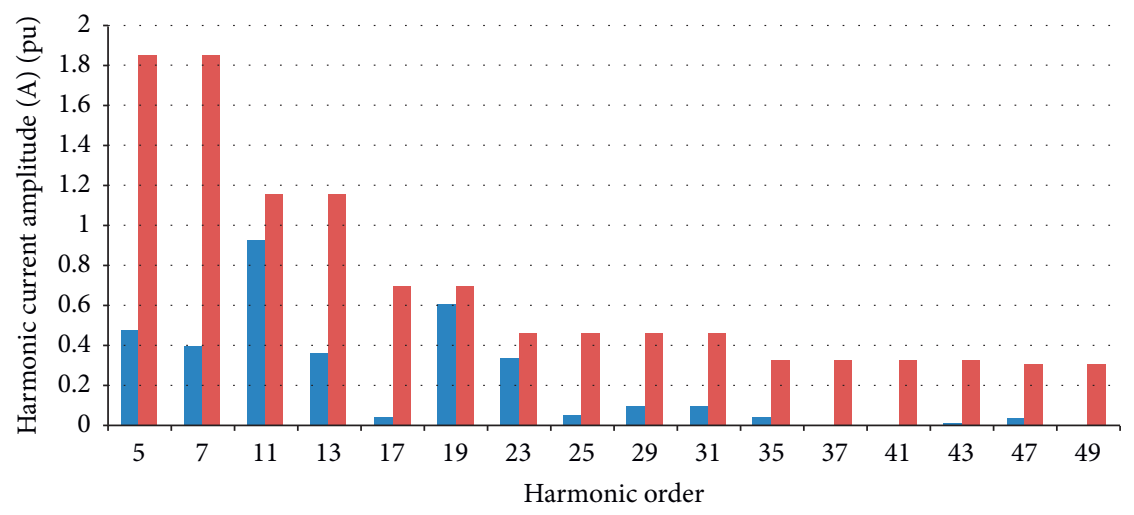

- IhLCL

- Ih_grid codes

FiguRE 12: Harmonic current spectrum with a theoretical LCL filter versus grid codes.

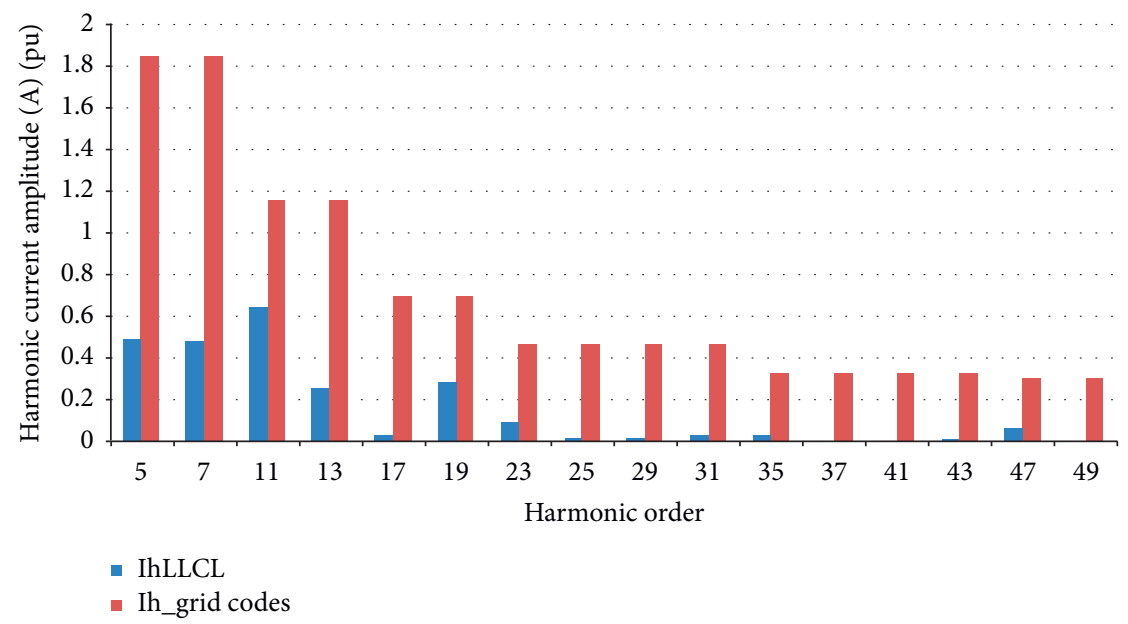

FIGURE 13: Harmonic current spectrum with a real-case LLCL filter versus grid codes.

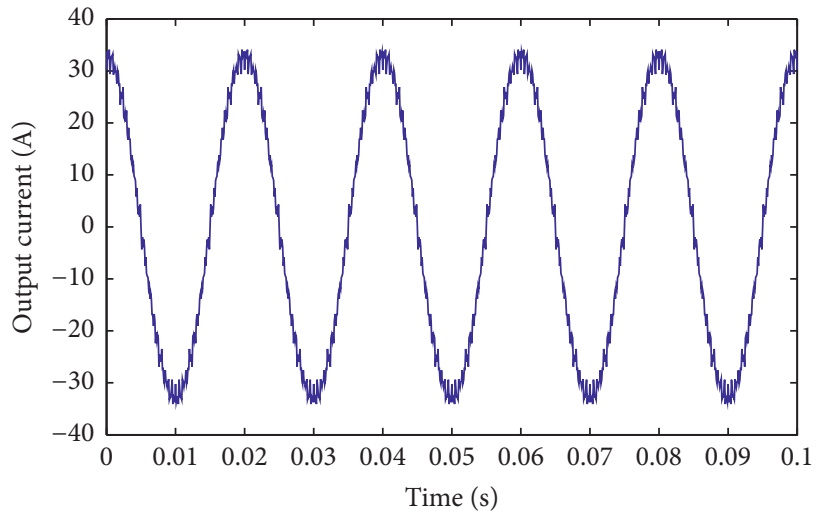

Figure 14: Obtained output current of the SHM-LCL strategy.

optimized SHM-LLCL filter is better than the SHM-LCL filter. This fact enhances that the SHM-LLCL is a very efficient strategy in terms of providing higher power quality and performances.
6.9. Frequency Domain Comparison between $L C L$ and LLCL Filters. Regardless LCL or LLCL [10], both filters combined with SHM respect successfully grid code requirements as shown in Figure 16. This appreciable result can be attributed 


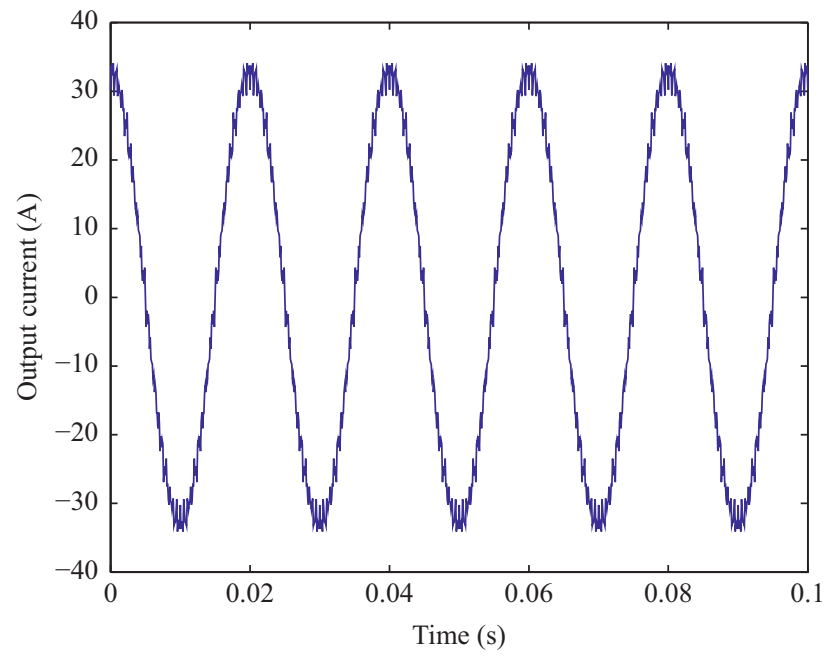

Figure 15: Obtained output current of the SHM-LLCL strategy.

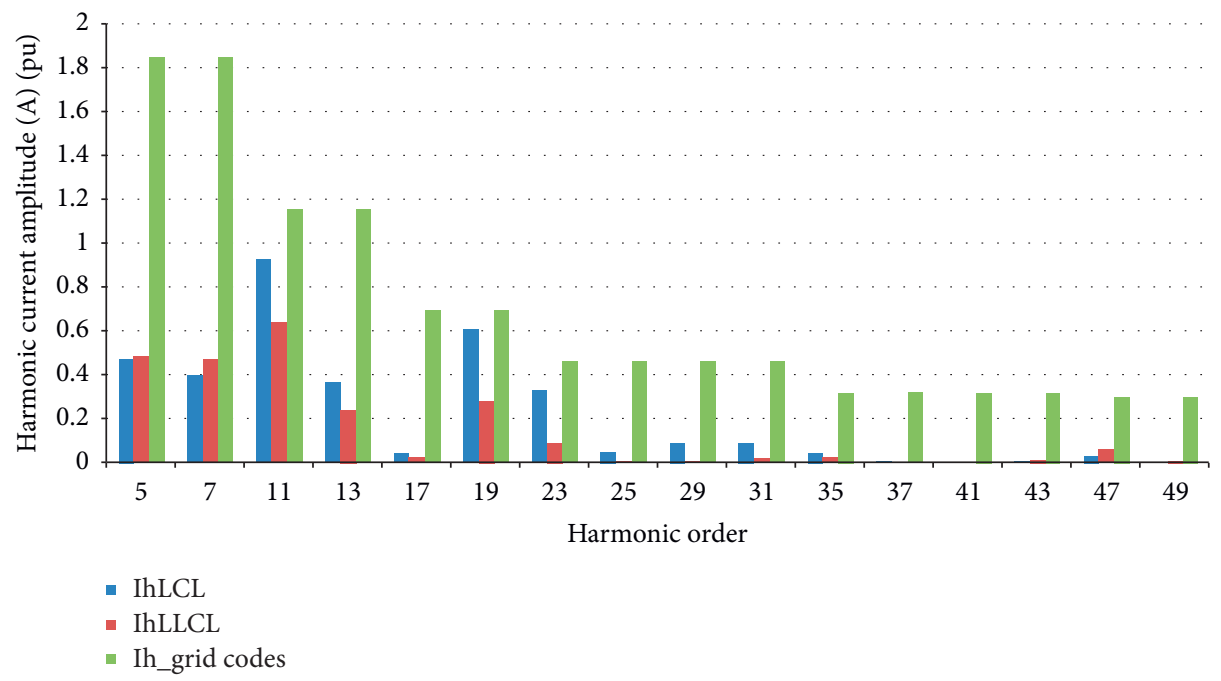

FIGURE 16: Harmonic current spectrum of LCL versus LLCL versus grid codes.

to the proper tuning, the stability margin of both filters, and the efficiency of SHM strategy.

The spectrum obtained with LLCL filters better than the LCL filter as almost harmonics amplitude is lower than the LCL filter. However, it is known and proved in previous works that the LLCL filter alone is less efficient than the LCL filter in terms of harmonics attenuation capability; but contrary to this expectation, Figure 16 shows that LLCL combined with SHM has better efficiency than the LCL filter. This result is obtained because the flexibility of the LLCL filter is valorized in our study by optimizing the tertiary cable length that impacts the antiresonance frequency and gives the advantage to LLCL filter with SHM strategy.

6.10. Influence of a Dead-Time Constraint on the Harmonic Current Spectrum. The addition of a dead-time constraint between two consecutive commutations of two IGBTs on the same arm has no effect on the optimization results. Indeed, with seven switching angles, we are with a relatively low switching frequency. For this, this constraint is easily respected. On the other hand, when the generated power is 
too low (too little sunshine), we intend to switch to a conventional synchronous PWM. In this case, this constraint will be taken into account, which means that the maximum commutation frequency should be less than 66 $\mathrm{kHZ}$.

The 1970s paper is the first paper where the relationship between switching angles and harmonics' rank is expressed under mathematical formulas regardless of the switching frequency or the semiconductor technologies.

Besides, in 1970, the calculators were not powerful enough to solve nonzero equations, that's why the author spoke only about elimination.

In 2000s paper, the author used the same mathematical equations, but nonzero equalities can be solved thanks to calculators' development.

Regarding the switching frequency, in our application, we will not exceed $1 \mathrm{kHz}$ with common silicon IGBT packs as we spoke in this study case about $1 \mathrm{MW}$-PV station. Otherwise, the radiated emission and power losses will be too high.

\section{Conclusion}

The present paper treats two sophisticated LCL and LLCL filter topologies, in order to meet grid code requirements. A special implementation has been achieved to highlight their efficiency. A comprehensive comparative analysis has been proved by various simulation results, showing that both filters successfully meet grid codes. Since we work with low switching frequency, the addition of a dead-time constraint has no effect on the optimization result.

This work shows that the optimized LLCL filter can provide higher quality and performances than LCL filter.

Finally, we have to confirm that luckily our suspected hypothesis is avoided. Fortunately, in our case, the harmonic current spectrum respected the standard limits, but this fact could not have been the case in another situation. This point has to be taken into account in the design phase.

\section{Data Availability}

No data were used to support this study.

\section{Conflicts of Interest}

The authors declare that they have no conflicts of interest.

\section{References}

[1] IEEE, IEEE Recommended Practice and Requirements for Harmonic Control in Electric Power Systems, IEEE Std 519-2014 (Revision of IEEE Std 519-1992), IEEE, Manhattan, NY, USA, 2014.

[2] Engineering Recommendation G5/4, Recommendation Planning Levels for Harmonic Voltage Distortion and the Connection of Non-linear-equipement to Transmission Systems and Distribution Networks in the United Kingdom, Engineering Recommendation G5/4, London, UK, 2001.

[3] Arrêté du, "Arrêté du 23 avril 2008 relatif aux prescriptions techniques de conception et de fonctionnement pour le raccordement à un réseau public de distribution d'électricité en basse tension ou en moyenne tension d'une installation de production d'énergie électrique (in French)," 2011.

[4] M. S. Chayjani and M. Monfared, "Design of LCL and LLCL filters for single-phase grid connected converters," IET Power Electronics, vol. 9, pp. 1-8, 2016.

[5] K. Arulkumar1, D. Vijayakumar2, and K. Palanisamy, "Design of optimal LLCL filter with an improved control strategy for single phase grid connected PV inverter," International Journal of Power Electronics and Drive System (IJPEDS), vol. 9, no. 1, pp. 114-125, 2018.

[6] W. Wu, Y. He, and F. Blaabjerg, "An LLCL power filter for single-phase grid-tied inverter," IEEE Transactions on Power Electronics, vol. 27, no. 2, 2012.

[7] H. S. Patel and R. G. Hoft, "Generalized techniques of harmonic elimination and voltage control in thyristor inverters: part I--Harmonic elimination," IEEE Transactions on Industry Applications, vol. 9, no. 3, pp. 310-317, 1973.

[8] L. G. Franquelo, J. Napoles, R. Portillo, J. I. Leon, and M. A. Aguirre, "A flexible selective harmonic mitigation technique to meet grid codes in three-level PWM converters," IEEE Transactions on Industrial Electronics, vol. 54, no. 6, pp. 3022-3029, 2007.

[9] J. Napoles, J. I. Leon, R. Portillo, L. G. Franquelo, and M. A. Aguirre, "Selective harmonic mitigation technique for high-power converters," IEEE Transactions on Industrial Electronics, vol. 57, no. 7, 2010.

[10] R. Chakroun, R. Ben Ayed, and N. Derbel, "Combined SHELLCL design for a real case photovoltaic power station," Journal of Control, Automation and Electrical Systems, vol. 64, pp. 1-9, 2020.

[11] K. Shi, "Non-fragile memory filtering of TS fuzzy delayed neural networks based on switched fuzzy sampled-data control," Fuzzy Sets and Systems, vol. 394, pp. 40-64, 2020.

[12] K. Shi, "Reliable asynchronous sampled-data filtering of T-S fuzzy uncertain delayed neural networks with stochastic switched topologies," Fuzzy Sets and Systems, vol. 381, pp. 1-25, 2020.

[13] X. Cai, "Robust H control for uncertain delayed TS fuzzy systems with stochastic packet dropouts," Applied Mathematics and Computation, vol. 385, Article ID 125432, 2020.

[14] C. Zhao, "Secure consensus of multi-agent systems with redundant signal and communication interference via distributed dynamic event-triggered control," ISA Transactions, vol. 112, pp. 89-98, 2020.

[15] S. Y. Hu, H. Huang, and D. Czarkowski, "Hybrid trigonometric differential evolution for optimizing harmonic distribution," in Proceedings of the IEEE International Symposium on Circuits and Systems, pp. 1306-1309, Kobe, Japan, May 2005.

[16] S. Y. Hu, H. Huang, and D. Czarkowski, "Fuzzy solution for approximating constrained optimal PWM using quantum evolutionary algorithm," in Chapter in Advances in Soft Computing: Soft Methodology and Random Information Systems, M. Lopez-Diaz, M. A. Gil, and P. Grzegorzewski, Eds., pp. 661-668, Springer-Verlag, Berlin, Germany, 2004. 